\title{
Estimation of Elastic Moduli of Particulate Composites by New Models and Comparison with Moduli Measured by Tension, Dynamic, and Ultrasonic Tests
}

\author{
G. Bourkas, I. Prassianakis, V. Kytopoulos, E. Sideridis, and C. Younis \\ Laboratory of Testing and Masterials, Department of Mechanics, Faculty of Applied Mathematical and Physical Sciences, \\ National Technical University of Athens, Zografou Campus, 15773 Athens, Greece \\ Correspondence should be addressed to C. Younis, cjyounis@central.ntua.gr
}

Received 29 January 2010; Revised 11 May 2010; Accepted 12 May 2010

Academic Editor: Paul Munroe

Copyright ( $) 2010$ G. Bourkas et al. This is an open access article distributed under the Creative Commons Attribution License, which permits unrestricted use, distribution, and reproduction in any medium, provided the original work is properly cited.

\begin{abstract}
The elastic constants of particulate composites are evaluated employing a theoretical cube-within-cube formation. Two new models of four and five components, respectively, formed by geometrical combination of three-component models existing in the literature, are used as Representative Volume Elements. Using the governing stress and strain equations of the proposed models, two new equations providing the static elastic and shear moduli of particulate composites are formulated. In order to obtain the dynamic elastic and shear moduli, the correspondence principle was applied successively to components connected in series and/or in parallel. The results estimated by the proposed models were compared with values evaluated from existing formulae in the literature, as well as with values obtained by tensile, dynamic, and ultrasonic experiments in epoxy/iron particulate composites. They were found to be close to values obtained by static and dynamic measurements and enough lower compared with values obtained from ultrasonic experiments. The latter is attributed to the high frequency of ultrasonics. Since measurements from ultrasonic's and from dynamic experiments depend on the frequency, the modulus of elasticity estimated by ultrasonic's is compared with that (storage modulus) estimated by dynamic experiments.
\end{abstract}

\section{Introduction}

Metal particles added in polymer matrices produce composite of greater density, improved electrical conductivity, better thermal conductivity, and consequently, improved behaviour at high operating temperature, and above all, highly improved mechanical properties. In general rigid fillers increase the elastic and shear moduli, and many theories have been developed to explain this effect.

Epoxy resins are the most suitable polymers for composite matrices and extensive research has been carried out on their rheological behavior [1-3] and their mechanical properties $[4,5]$.

A rigorous description of a composite system consisting of a matrix in which filler particles have been dispersed is not an easy task. In fact, a great number of geometrical, topological, and mechanical parameters are necessary, the majority of which varies statistically or is simply unknown.
Theoretical treatments usually attempt to exploit as much as possible readily available information, which in most cases consists of the mechanical properties of the matrix and the filler and the volume fraction of the latter, while suitable assumptions cover missing data. The best approximation appears to be the determination of upper and lower bounds for the effective moduli of the composite, based on variational principles of mechanics, developed by Hashin [6].

Analytical solutions are valid up to some fairly low filler volume fraction as they have to ignore, for reasons of efficiency, any mechanical interaction between neighbouring inclusions [7]. Referring in particular to the moduli, the existing expressions are resulted from the elasticity theory or express a kind of law of mixtures or are simply an attempt to match theoretical curves to experimental data [8-22]. In most of them a perfect adhesion between matrix and filler was assumed as existing between phases of the composite. 


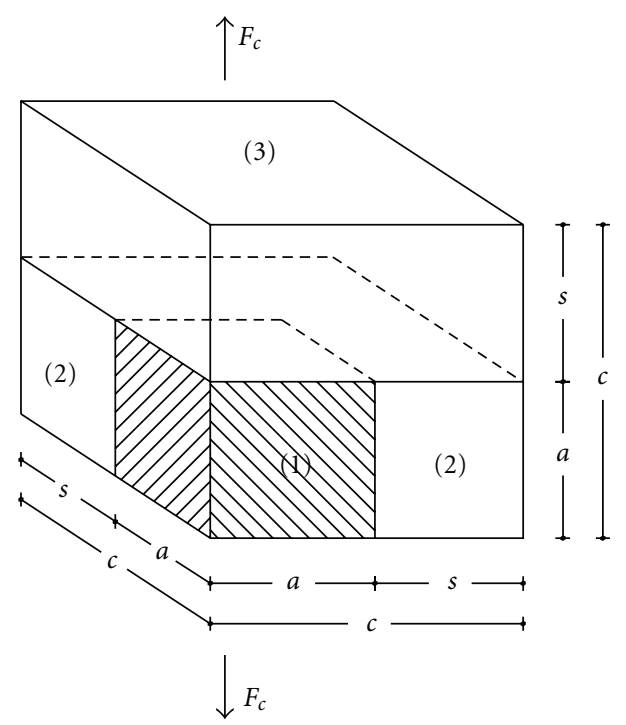

(a)

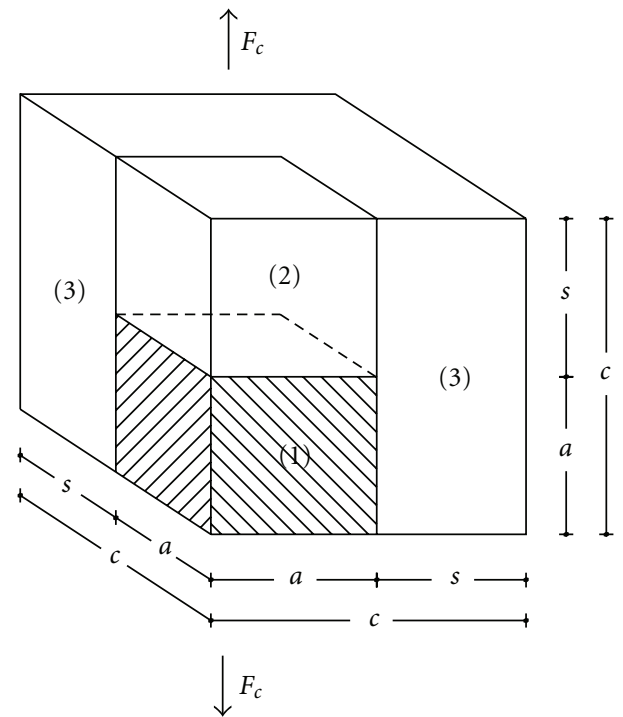

(b)

Figure 1: The three-part models. (a) Paul model, model 1, (b) Issay-Cohen model, model 2.

In [23] the shear moduli of particulate composites are obtained by means of two cube-within-cube models. In [24] a concept of the interaction between the fillers using different distribution of the inclusions into the volume of the matrix is presented. In [25-28] the effect of the interphase on the values of the elastic modulus of the particulate composites is examined.

There is also a sizeable volume of literature dealing with dynamic behaviour of heterogeneous systems, where the dispersed phase is a relatively rigid inclusion [29-32]. In [6] a correspondence principle is developed, by means of which effective complex moduli of viscoelastic composites can be determined on the basis of analytical expressions for effective elastic moduli of composites. It is generally found that the storage modulus increases by increasing frequency,

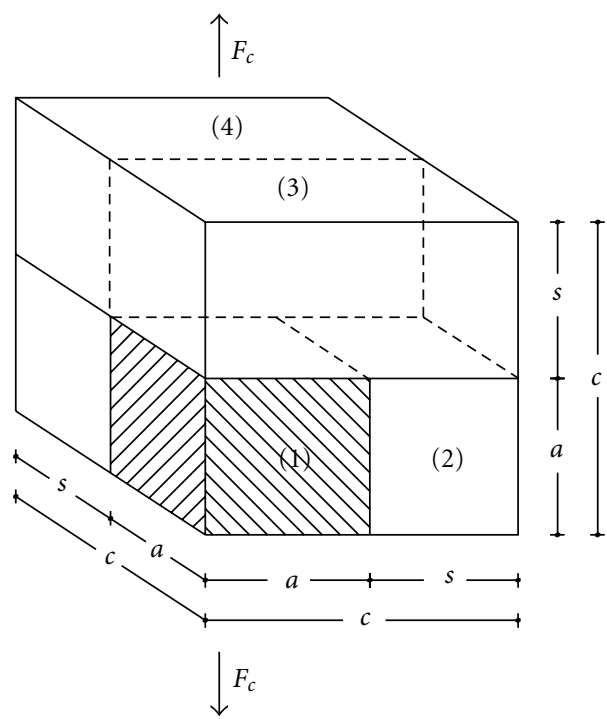

(a)

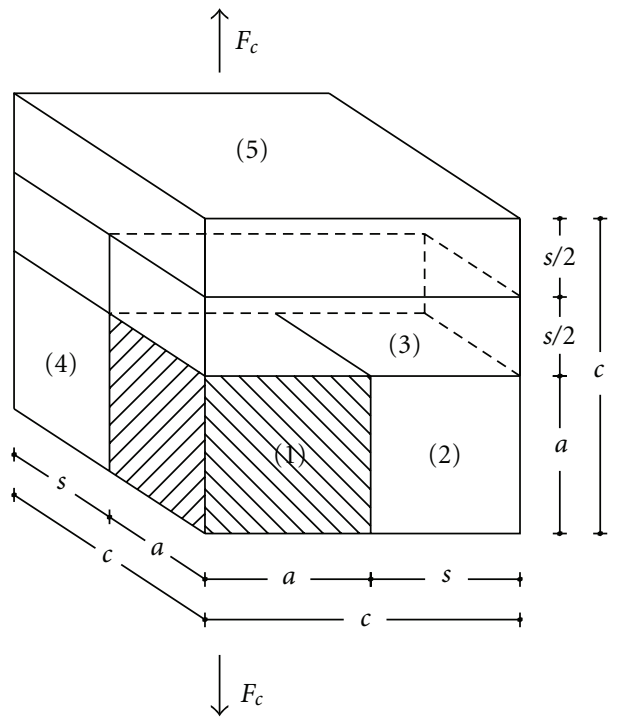

(b)

FIGURE 2: The proposed models. (a) Four-part model, model 3, (b) Five-part model, model 4.

for constant temperature, where for high frequency values tend to the value of static elastic modulus of the particulate composite.

In this paper by considering the cube-within-cube formation the elastic constants of particulate-filled composites, by using two models of four and five parts, were evaluated. These models are considered geometrically hybridique in relation with the three-part models existing in the literature [18-20]. The assumptions of [18-23] were used, for the evaluation of the static elastic and shear moduli. According to [6] the correspondence principle has been used for the prediction of the dynamic elastic constants of the particulate composite. Tensile experimental results, dynamic results, and results from ultrasonic tests in epoxy/iron particulate composites were compared to the derived theoretical results. 


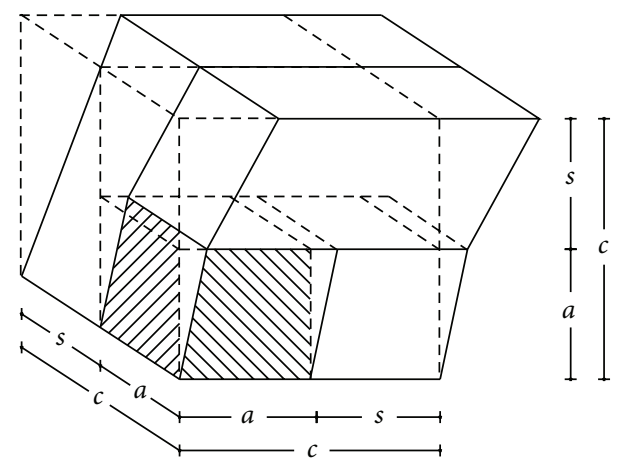

(a)

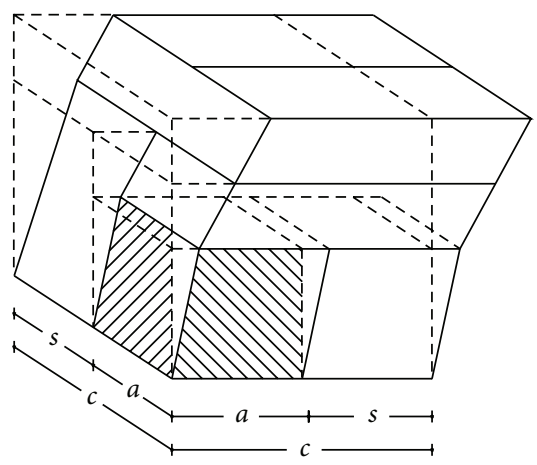

(b)

Figure 3: The proposed models in state of shear deformation. (a) model 3, (b) model 4.

Also theoretical results predicted from existing models in the literature, whose equations are in Appendices A and $\mathrm{B}$, were used for comparison. In order to investigate the effect of frequency, the results obtained from ultrasonic experiments were compared with those obtained from dynamic experiments carried out in epoxy/iron particulate composites, since frequency is a common characteristic.

\section{Theoretical Considerations}

The theoretical analysis will be based on the following assumptions.

(i) Particles are perfectly cubic in shape.

(ii) The distribution of the matrix volume to each individual inclusion is also perfectly cubic in shape. The respective sides of two cubes are parallel.

(iii) There are many filler particles and their distribution is uniform, so that the composite may be regarded as a quasihomogeneous and isotropic material.

(iv) The matrix and the filler are elastic, isotropic, and homogeneous.

(v) The volume fraction of filler is sufficiently small for the interaction between fillers to be neglected.

(vi) The deformation applied to the composite is small enough to maintain linearity of the stress-strain relations. (vii) There is no transverse variation of the strains in the components which are connected in parallel and have the same length in the load direction.

(viii) The stresses do not vary in the direction of the applied load in the components which are connected in series and have the same cross-sections.

\subsection{Static Elastic Constants}

2.1.1. Elastic Moduli. According to [18-20], the models shown in Figures 1(a) and 1(b), named model 1 and 2 respectively, are three-part composites. From Figure 1 the filler volume fraction is given by

$$
v_{f}=\frac{\alpha^{3}}{c^{3}} .
$$

For uniaxial load in the figures direction, their elastic moduli are given, respectively, by [18-20]

$$
\begin{aligned}
& E_{c}^{(1)}=E_{m}\left(\frac{1+(m-1) v_{f}^{2 / 3}}{1+(m-1)\left(v_{f}^{2 / 3}-v_{f}\right)}\right), \\
& E_{c}^{(2)}=E_{m}\left(1+\frac{v_{f}}{m /(m-1)-v_{f}^{1 / 3}}\right),
\end{aligned}
$$

where $m=E_{f} / E_{m}$ and $E_{f}, E_{m}$, and $E_{c}$ are the elastic moduli of the filler, matrix and composite, respectively. The indices (1) and (2) refer to model 1 and 2, respectively.

The model presented in Figure 2(a) according to [1820] named model 3 , for uniaxial loading in the figure direction, has four components. The components (1) and (2) are in parallel, and both are in series with component (3). The above components are in parallel with component (4). For uniaxial loading in Figure 2(a) direction, from forces equilibrium and elongations equality, we can write the following equations:

$$
\begin{gathered}
\sigma_{c}=\sigma_{3} v_{f}^{1 / 3}+\sigma_{4}\left(1-v_{f}^{1 / 3}\right), \\
\sigma_{3} v_{f}^{1 / 3}=\sigma_{1} v_{f}^{2 / 3}+\sigma_{2}\left(v_{f}^{1 / 3}-v_{f}^{2 / 3}\right), \\
\varepsilon_{1}=\varepsilon_{2}, \\
\varepsilon_{4}=\varepsilon_{c}, \\
\varepsilon_{c}=\varepsilon_{1} v_{f}^{1 / 3}+\varepsilon_{3}\left(1-v_{f}^{1 / 3}\right),
\end{gathered}
$$

where the indices $1,2,3,4$, and $c$ correspond to the parts (1), (2), (3), and (4) and the composite, respectively.

The constitutive equations are given from the Hooke law as follows:

$$
\begin{gathered}
\sigma_{1}=\varepsilon_{1} E_{f}, \\
\sigma_{j}=\varepsilon_{j} E_{m}, \quad j=2,3,4, \\
\sigma_{c}=\varepsilon_{c} E_{c} .
\end{gathered}
$$




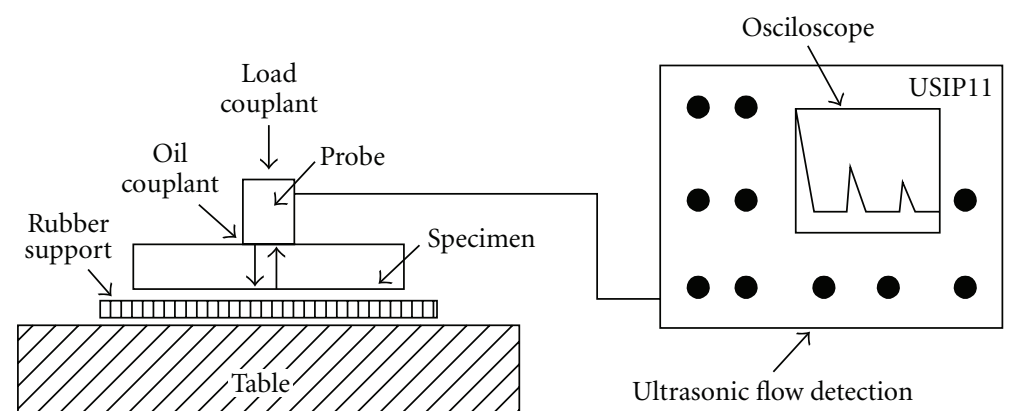

FIGURE 4: Schematic diagram of the used ultrasonic pulse-echo measuring system.

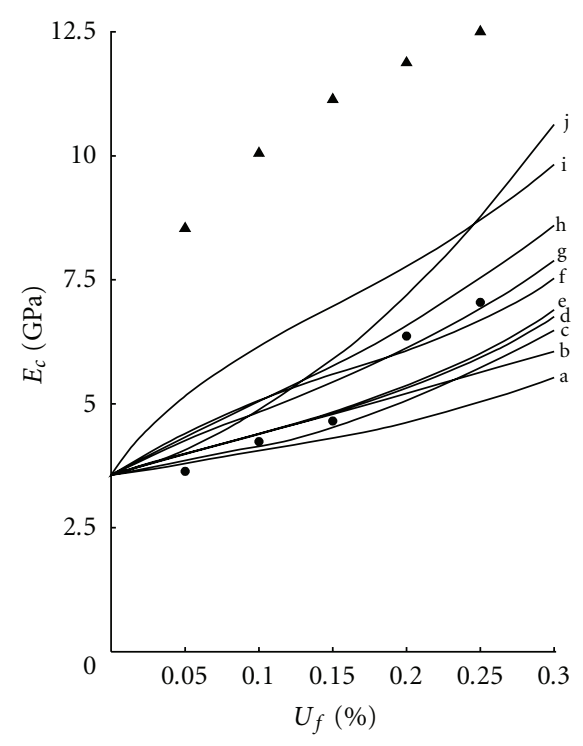

Experimental points

- Tension measurements

- Ultrasonic measurements

FIGURE 5: Elastic modulus versus filler in iron/epoxy particulate composites volume fraction. (a) Equations (A.5) and (A.6), (b) equation (A.1), (c) equation (3), (d) equation (A.4), (e) equation (A.3), (f) equation (A.11), (g) equation (12), (h) equations (17), (i) equation (2), and (j) equation (A.2).

By combining (4)-(11) the following expression for the elastic modulus of particulate composite is obtained:

$$
E_{c}^{(3)}=E_{m}\left(1+\frac{v_{f}}{1 /(m-1)+v_{f}^{1 / 3}-v_{f}^{2 / 3}}\right),
$$

where $m=E_{f} / E_{m}$.

Considering now the model presented in Figure 2(b) called model 4 one can observe that it consists of five components and that the coupling of components (1), (2), (3) and (4) is the same as in the case of model 1. The element consisting of these four components is connected in series with component (5). For uniaxial load along the direction

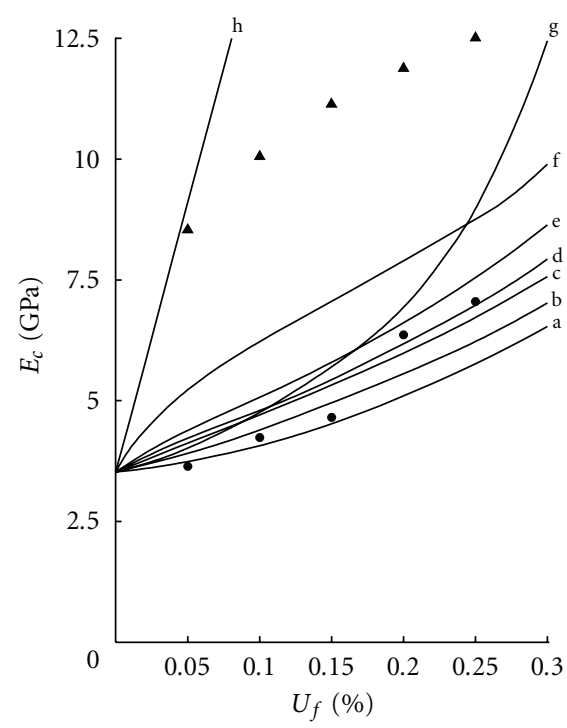

Experimental points

- Tensile experimental values

- Ultrasonic measurements

Figure 6: Elastic modulus versus filler content in iron/epoxy particulate composites. (a) Equation (3), (b) equation (A.9), (c) upper bound, equation (A.7), (d) equation (12), (e) equations (17), (f) equation (2), (g) equation (A.8), and (h) equation (A.10).

shown in Figure 2(b) the force equilibrium and the strain compatibility give the following equations

$$
\begin{gathered}
\sigma_{c}=\sigma_{5}, \\
\sigma_{c}=\sigma_{3} v_{f}^{1 / 3}+\sigma_{4}\left(1-v_{f}^{1 / 3}\right), \\
\sigma_{3} v_{f}^{1 / 3}=\sigma_{1} v_{f}^{2 / 3}+\sigma_{2}\left(v_{f}^{1 / 3}-v_{f}^{2 / 3}\right), \\
\varepsilon_{1}=\varepsilon_{2}, \\
2 \varepsilon_{c}=\varepsilon_{4}\left(1+v_{f}^{1 / 3}\right)+\varepsilon_{5}\left(1-v_{f}^{1 / 3}\right), \\
\varepsilon_{4}\left(1+v_{f}^{1 / 3}\right)=2 \varepsilon_{1} v_{f}^{1 / 3}+\varepsilon_{3}\left(1-v_{f}^{1 / 3}\right) .
\end{gathered}
$$




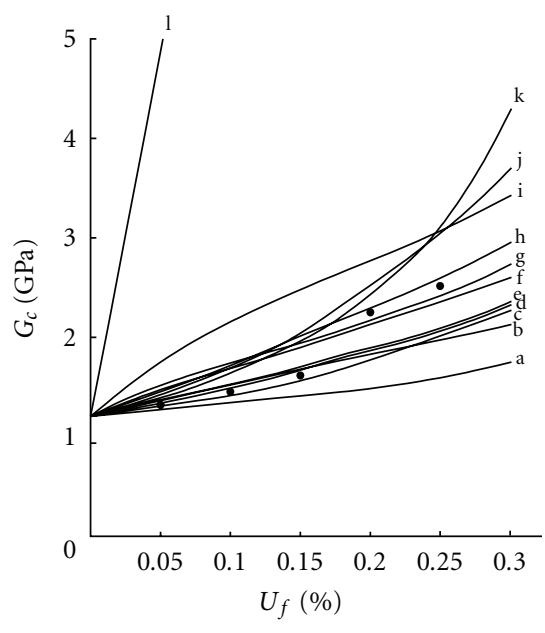

Experimental points

- Tensile tests

FIGURE 7: Shear modulus via filler content in iron/epoxy particulate composites. (a) The inverse law of mixtures, (b) equation (A.1), (c) equation (19), (d) equation (A.3), (e) equation (A.4), (f) equation (A.11), (g) equation (20), (h) equations (21) and (22), (i) equation (18), (j) equation (A.2), (k) equation (A.8), and (1) law of mixtures.

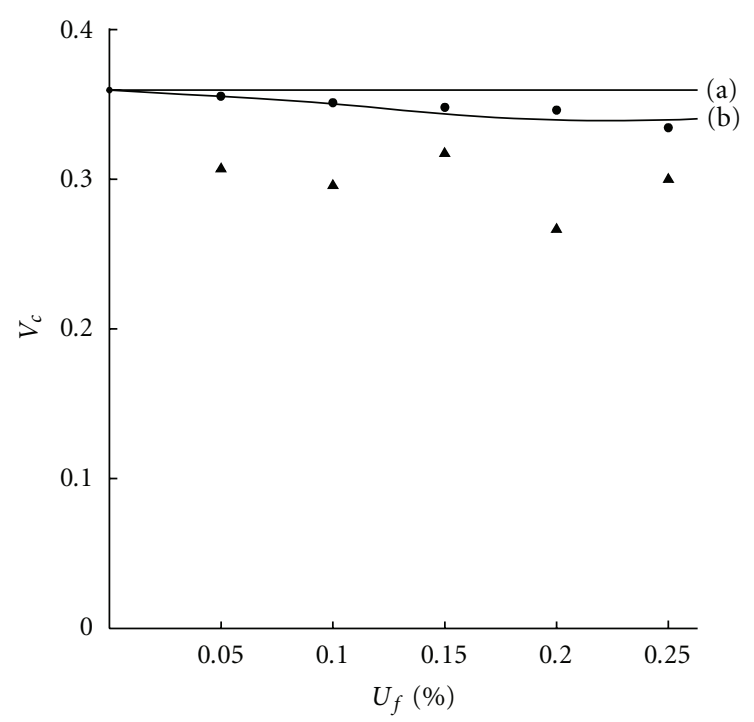

Experimental points

- Tensile experiments

(a) Equation (23)

- Ultrasonic measurements

(b) Equation (24)

Figure 8: Poisson ratio versus filler content in iron/epoxy particulate composites. (a) Equation (24), (b) equation (23).

The constitutive equations are given from Hook's law

$$
\begin{gathered}
\sigma_{1}=\varepsilon_{1} E_{f}, \\
\sigma_{j}=\varepsilon_{j} E_{m} \quad j=2,3,4,5, \\
\sigma_{c}=\varepsilon_{c} E_{c} .
\end{gathered}
$$

For the case of model 4 which is evaluated using the superposition principle, the following constitutive equation is also used:

$$
\sigma_{a}=\varepsilon_{a} E_{a}
$$

where index $a$ corresponds to the composite consisting of components 1, 2, and 3 of model 4 with

$$
\begin{aligned}
\sigma_{a} & =\sigma_{3}, \\
\varepsilon_{a} & =\varepsilon_{4} .
\end{aligned}
$$

Solving for $E_{a}$ and $E_{c}$ the respective equation of the model one obtains

$$
\begin{gathered}
E_{c}^{(4)}=E_{m} \cdot\left(1+\frac{2 v_{f}^{2 / 3}}{\left(m v_{f}^{1 / 3}+1\right) /(m-1)-v_{f}^{2 / 3}}\right), \\
E_{c}^{(4)}=E_{m}\left(1+\frac{v_{f}^{1 / 3}+v_{f}^{2 / 3}}{2 /(n-1)+v_{f}^{1 / 3}-v_{f}^{2 / 3}}\right),
\end{gathered}
$$

where $m=E_{f} / E_{m}$, and $n=E_{\alpha} / E_{m}$.

2.1.2. Shear Moduli. In [23] models 1 and 2 under shear loading are presented in state of deformation. According to [23] models 3 and 4 in state of deformation under shear loading in the figure directions are presented in Figures 3(a) and 3(b). The couplings remain the same as in the case of uniaxial loading in Figures 2(a) and 2(b). The same remark has been made for models 1 and 2 in [23]. Thus, analogous equations for the shear, as in the case of uniaxial loading are obtained. From this fact for the shear moduli identical equations are founded as in the case of elastic modulus where in the place of $E_{m}, E_{f}$, and $E_{c}, G_{m}, G_{f}$, and $G_{c}$, respectively, are placed, where $G$ is the shear modulus. Therefore, it follows that

$$
\begin{gathered}
G_{c}^{(1)}=G_{m}\left(\frac{1+(\rho-1) v_{f}^{2 / 3}}{1+(\rho-1)\left(v_{f}^{2 / 3}-v_{f}\right)}\right), \\
G_{c}^{(2)}=G_{m}\left(1+\frac{v_{f}}{\rho /(\rho-1)-v_{f}^{1 / 3}}\right),
\end{gathered}
$$

$$
G_{c}^{(3)}=G_{m}\left(1+\frac{v_{f}}{1 /(\rho-1)+v_{f}^{1 / 3}-v_{f}^{2 / 3}}\right),
$$

$$
G_{a}^{(4)}=G_{m}\left(1+\frac{2 v_{f}^{2 / 3}}{\left(\rho \cdot v_{f}^{1 / 3}+1\right) /(\rho-1)-v_{f}^{2 / 3}}\right),
$$

$$
G_{c}^{(4)}=G_{m}\left(1+\frac{v_{f}^{1 / 3}+v_{f}^{2 / 3}}{2 /(q-1)+v_{f}^{1 / 3}-v_{f}^{2 / 3}}\right),
$$

where $\rho=G_{f} / G_{m}$ and $q=G_{a}^{(4)} / G_{m}$.

For comparison, from the existing equations in the literature (A.1), (A.2), (A.3), (A.4), (A.8), and (A.11) are 


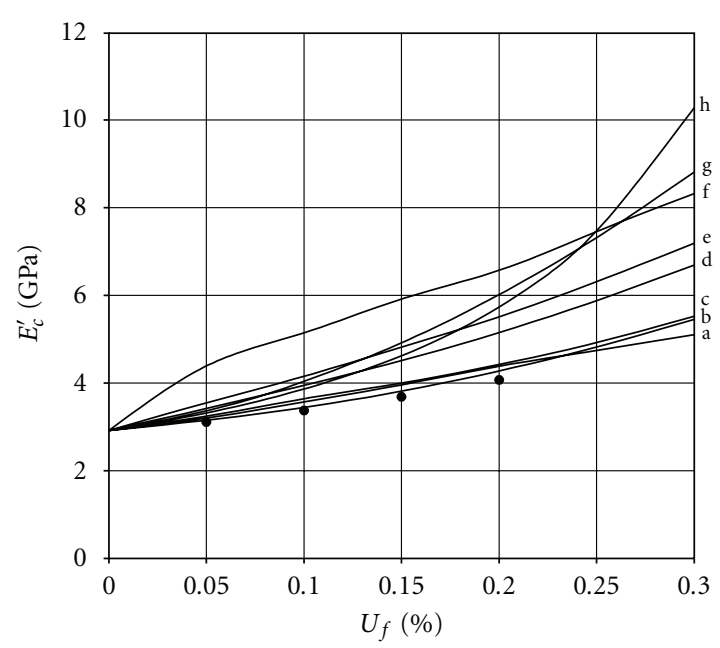

- Experimental points

FIGURE 9: The storage modulus $E_{c}^{\prime}$ versus filler volume fraction for frequency $f=0,1 \mathrm{~Hz}$ in epoxy/iron particulate composite. (a) equation (B.1), (b) model 2, equations (28) and (30), (c) eq(B.5), (d) model 3, equations (28) and (30), (e) model 4, equations (28) and (30), (f) model 1, equations (28) and (30), (g) equation (B.3), and (h) equation (B.7).

examined where in the place of $E_{c}, E_{m}$, and $E_{f}, G, G_{m}$, and $G_{f}$, respectively, are placed.

2.1.3. Poisson Ratios. The Poisson ratios can be evaluated from the following two different modes and then by comparing the theoretical results among each other and with experimental results.

(a) Considering the particulate composite as homogeneous and isotropic material and having evaluated as previously the respective elastic and shear moduli, the Poisson ratio of each model may be found from the relation

$$
G_{c}=\frac{E_{c}}{2\left(v_{c}+1\right)}
$$

(b) From the inverse law of mixture, which seems to fit fairly well the experiment, for the case of particulate composites [25], we write

$$
\frac{1}{v_{c}}=\frac{v_{f}}{v_{f}}+\frac{v_{m}}{v_{m}}
$$

\subsection{Dynamic Elastic Constants}

2.2.1. Dynamic Elastic Moduli. Models 1, 2, 3, and 4 consist of components connected in parallel and/or in series. Consequently the static elastic and shear moduli could also be obtained by successive application of the law of mixtures and the inverse law of mixtures. This procedure

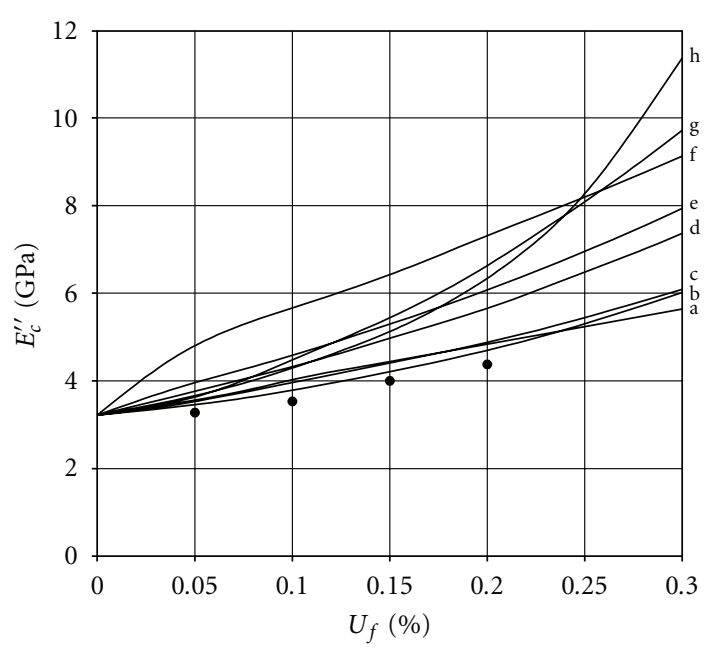

- Experimental points

Figure 10: The loss modulus $E_{c}^{\prime \prime}$ versus filler volume fraction for frequency $f=0,1 \mathrm{~Hz}$ in epoxy/iron particulate composite. (a) equation (B.1), (b) model 2, equations (28) and (30), (c) eq(B.5), (d) model 3, equations (28) and (30), (e) model 4, equations (28) and (30), (f) model 1, equations (28) and (30), (g) equation (B.3), and (h) equation (B.7).

is used for the evaluation of the dynamic elastic and shear moduli.

Considering a two-component composite, the complex modulus $E_{j}^{*}$ of each component is given by

$$
E_{j}^{*}=E_{j}^{\prime}+i E_{j}^{\prime \prime}
$$

where $j=1,2, E_{j}^{\prime}$ is the storage modulus, and $E_{j}^{\prime \prime}$ is the loss modulus.

According to the correspondence principle when these two components are connected in parallel it comes out that

$$
E_{1,2}^{*}=E_{1}^{*} v_{1}+E_{2}^{*} v_{2}
$$

where $v_{1}, v_{2}$ are the volume fractions of components (1) and (2), and $E_{1}^{*}, E_{2}^{*}$ and $E_{1,2}^{*}$ are the complex moduli of components (1) and (2) and the two-component composite, respectively, and

$$
E_{1,2}^{*}=E_{1,2}^{\prime}+i E_{1,2}^{\prime \prime}
$$

From (25), (26), and (27) it comes out that

$$
\begin{gathered}
E_{1,2}^{\prime}=E_{1}^{\prime} v_{1}+E_{2}^{\prime} v_{2}, \\
E_{1,2}^{\prime \prime}=E_{1}^{\prime \prime} v_{1}+E_{2}^{\prime \prime} v_{2},
\end{gathered}
$$

where $E_{1,2}^{\prime}$ and $E_{1,2}^{\prime \prime}$ are the storage modulus and the loss modulus of the two-component composites. 


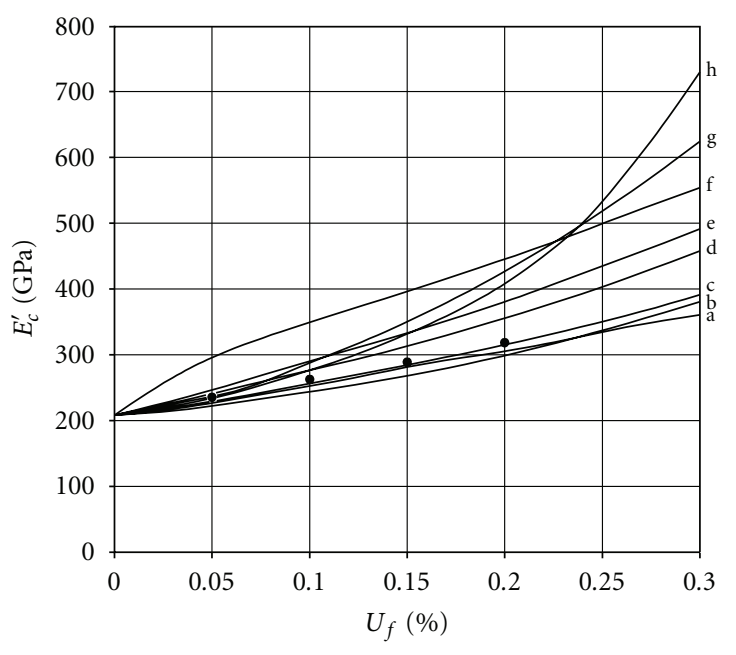

- Experimental points

Figure 11: The storage modulus $E^{\prime}{ }_{c}$ versus filler volume fraction for frequency $f=50 \mathrm{~Hz}$ in epoxy/iron particulate composite. (a) equation (B.1), (b) model 2, equations (28) and (30), (c) eq(B.5), (d) model 3, equations (28) and (30), (e) model 4, equations (28) and (30), (f) model 1, equations (28) and (30), (g) equation (B.3), (h) equation (B.7).

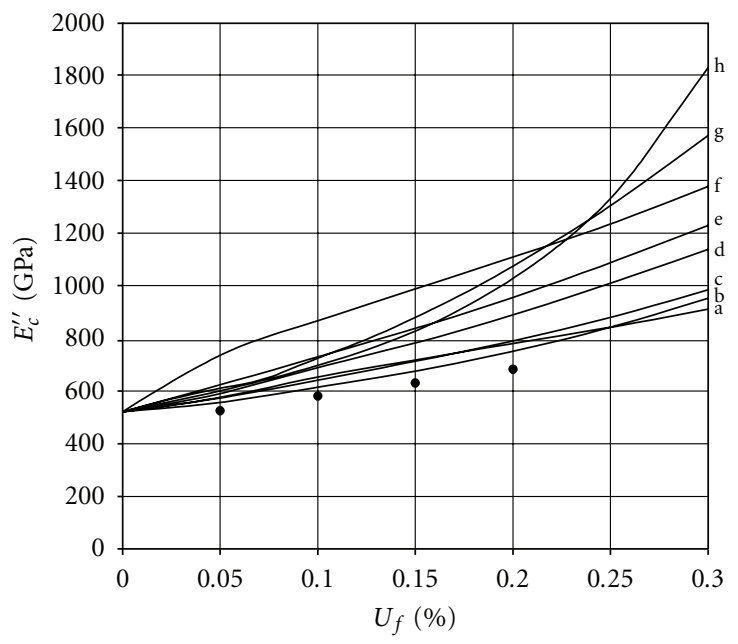

- Experimental points

Figure 12: The loss modulus $E_{c}^{\prime \prime}$ versus filler volume fraction for frequency $f=50 \mathrm{~Hz}$ in epoxy/iron particulate composite. (a) Equation (B.1), (b) model 2, equations (28) and (30), (c) eq(B.5), (d) model 3, equations (28) and (30), (e) model 4, equations (28) and (30), (f) model 1, equations (28) and (30), (g) equation (B.3), and (h) equation (B.7).

Using the correspondence principle when the components of a two-component composite are connected in series, one obtains
Introducing (25) and (26) into (29) one obtains

$$
\begin{gathered}
\tan \delta_{1,2}=\frac{E_{1}^{\prime \prime}\left({E_{2}^{\prime 2}}^{2}+{E_{2}^{\prime \prime}}^{2}\right) v_{1}+E_{2}^{\prime \prime}\left({E_{1}^{\prime 2}}^{2}+{E_{2}^{\prime 2}}^{2}\right) v_{2}}{E_{1}^{\prime}\left({E_{2}^{\prime 2}}^{2}+{E_{2}^{\prime \prime}}^{\prime 2}\right) v_{1}+E_{2}^{\prime}\left({E_{1}^{\prime 2}}^{2}+{E_{2}^{\prime 2}}^{2}\right) v_{2}} \\
\frac{1}{E_{1,2}^{\prime}\left(1+\tan \delta_{1,2}\right)}=\frac{E_{1}^{\prime} v_{1}}{{E_{1}^{\prime}}^{2}+E_{1}^{\prime \prime 2}}+\frac{E_{2}^{\prime} v_{2}}{{E_{1}^{\prime 2}}^{2}+E_{1}^{\prime \prime 2}}, \\
\tan \delta_{1,2}=\frac{E_{1,2}^{\prime \prime}}{E_{1,2}^{\prime}}
\end{gathered}
$$

from which one obtains $E_{1,2}^{\prime}, E_{1,2}^{\prime \prime}$, where $\delta_{1,2}$ is the phase lag between strain and stress.

The dynamic modulus of the composite consisting of a number components, as in the presented models, can now be obtained by a successive application of (28) and (30).

2.2.2. Dynamic Shear Moduli. As in the case of the static elastic moduli, the dynamic shear moduli can be obtained from (28) and (30) by replacing $E_{1}^{\prime}, E_{1}^{\prime \prime}, E_{2}^{\prime}, E_{2}^{\prime \prime}, E_{1,2}^{\prime}$ and $E_{1,2}^{\prime \prime}$ by $G_{1}^{\prime}, G_{1}^{\prime \prime}, G_{2}^{\prime}, G_{2}^{\prime \prime}, G_{1,2}^{\prime}$, and $G_{1,2}^{\prime \prime}$, respectively.

2.2.3. Dynamic Poisson Ratios. Since in the most polymers the same phase lag is observed in tension and shear, one has

$$
\frac{E_{m}^{\prime \prime}}{E_{m}^{\prime}}=\frac{G_{m}^{\prime \prime}}{G_{m}^{\prime}}
$$

Applying the correspondence principle in the equation

$$
G_{m}=\frac{E_{m}}{2\left(1+v_{m}\right)}
$$

with

$$
\begin{aligned}
v_{m}^{*} & =v_{m}^{\prime}-i v_{m}^{\prime \prime}, \\
G_{m}^{*} & =G_{m}^{\prime}+i G_{m}^{\prime \prime}, \\
E_{m}^{*} & =E_{m}^{\prime}+i E_{m}^{\prime \prime},
\end{aligned}
$$

it comes out that

$$
\begin{gathered}
v_{m}^{\prime}=\frac{E_{m}^{\prime} G_{m}^{\prime}+E_{m}^{\prime \prime} G_{m}^{\prime \prime}}{2\left(G_{m}^{\prime 2}+G_{m}^{\prime \prime 2}\right)}-1, \\
v_{m}^{\prime \prime}=0 .
\end{gathered}
$$

The dynamic Poisson ratios of the composite are now obtained applying the correspondence principle into equation

$$
G_{c}=\frac{E_{c}}{2\left(1+v_{c}\right)}
$$

which gives

$$
v_{c}^{*}=\frac{E_{c}^{*}}{2 E_{c}^{*}}-1
$$


from which one obtains

$$
\begin{gathered}
v_{c}^{\prime}=\frac{E_{c}^{\prime} G_{c}^{\prime}+E_{c}^{\prime \prime} G_{c}^{\prime \prime}}{2\left(G_{c}^{\prime 2}+G_{c}^{\prime 2}\right)}-1, \\
v_{c}^{\prime \prime}=\frac{E_{c}^{\prime} G_{c}^{\prime \prime}-E_{c}^{\prime \prime} G_{c}^{\prime}}{2\left(G_{c}^{\prime 2}+{G_{c}^{\prime \prime}}^{2}\right)} .
\end{gathered}
$$

When the inverse law of mixture is considered for the Poisson ratio of the composite it comes out that

$$
\begin{gathered}
\frac{1}{v_{c}^{\prime}}=\frac{v_{f}}{v_{f}}+\frac{v_{m}}{v_{m}^{\prime}}, \\
v_{c}^{\prime \prime}=0
\end{gathered}
$$

which gives

$$
\frac{E_{c}^{\prime \prime}}{E_{c}^{\prime}}=\frac{G_{c}^{\prime \prime}}{G_{c}^{\prime}} .
$$

2.3. Ultrasonic Equipment and Measurement Procedures. Energy pulse propagation through the structure at frequencies above the audible range can be related to the material properties. The velocity propagation can be measured since modulus $=$ density $\times(\text { velocity })^{2}$. However, the main aim of the ultrasonic testing of materials which contain discontinuities is to determine the effects of interaction between sound waves and material properties. The basic parameters required for all ultrasonic measuring methods are sound velocities and sound attenuation through the material in which the sound wave travels. Sound velocities $c_{\ell}$ and $c_{t}$ of the longitudinal and transverse waves, respectively, and the density $\rho_{c}$ of the material are used for the evaluation of the elastic modulus $E_{c}$, the Poisson ratio $v_{c}$, and the shear modulus $G_{c}$ via he following relationships:

$$
\begin{gathered}
E_{c}=\frac{\left(1+v_{c}\right)\left(1-2 v_{c}\right)}{\left(1-v_{c}\right)} \rho_{c} \cdot c_{\ell}^{2}, \\
v_{c}=\frac{1 / 2\left(c_{\ell} / c_{t}\right)^{2}-1}{\left(c_{\ell} / c_{t}\right)^{2}-1}, \\
G_{c}=\rho_{c} c_{t}^{2} .
\end{gathered}
$$

Figure 4 shows a schematic diagram of the ultrasonic pulseecho measuring system used. The system consists of a broad band $(0.5-15 \mathrm{MHz})$ ultrasonic pulser-receiver flaw detector (Krautkramer) which can generate and receive electric pulses up to $15 \mathrm{MHz}$. K2G and $\mathrm{K} 2 \mathrm{~N}$ probes were used as transmitting-receiving transducers of sound waves, producing ultrasounds of 2 and $4 \mathrm{MHz}$, respectively. A simple machine oil was used as the transducer/specimen interface couplant. A contact load for both probes of $9.88 \mathrm{~N}$ was applied to the transducer/specimen interface.

The pulser section produces and injects ultrasonic pulses into the specimen through the transducer, and the reflected signals produced are amplified by the receiver section of the equipment and displayed on the oscilloscope.

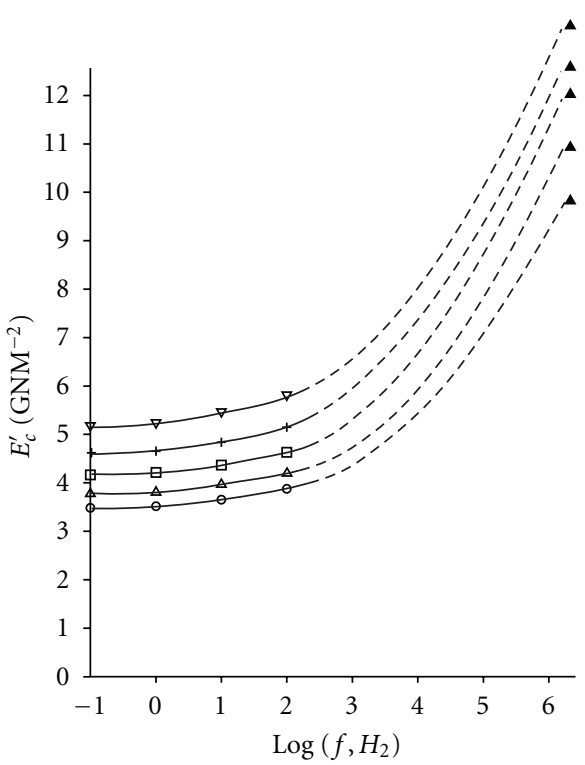

Experimental points (dynamic tests)

$\circ 0.05$ volume $(\%)$

$\triangle 0.1$ volume $(\%)$

0.15 volume $(\%)$

+0.2 volume $(\%)$

$\nabla 0.25$ volume (\%)

- Ultrasonic measurements

Figure 13: Variation of dynamic storage modulus of composite $E_{c}^{\prime}$ versus frequency for different values of filler volume fraction.

The sound velocity $c_{\ell}^{*}$ of the longitudinal waves of each specimen was evaluated using the relationship

$$
c_{\ell}^{*}=c_{\ell} \frac{d_{x}}{d_{y}},
$$

where $c_{\ell}$ is the sound velocity of the reference block, $d_{x}$ is the real specimen thickness, and $d_{g}$ is the equivalent thickness of the specimen, which is measured on the screen of the oscilloscope.

\section{Material and Experimental Work}

3.1. Testing Material in Tension and Ultrasonic Measurements. The specimen used consisted of a matrix material, which was a cold setting system based on a diglycidyl ether of bisphenol. A resin having an epoxy equivalent of 185-192, a viscosity of $15 \mathrm{Nsm}^{-2}$ at $25^{\circ} \mathrm{C}$, and molecular mass between 370 and 384 , was cured with $8 \mathrm{wt}-\%$ triethylenetetramine filled with iron particles of average radius $75 \mu \mathrm{m}$. The elastic moduli of the matrix and filler were 3,5 , and $210 \mathrm{GNm}^{-2}$, respectively, whereas the Poisson ratios were 0, 36, and 0, 29, respectively.

3.1.1. Tensile Experiments. Dogbone specimens with constant dimensions of measuring area $6 \times 3 \mathrm{~mm}$ and length $45 \mathrm{~mm}$ were used during the tensile tests which were carried out with an Instron type testing machine at room temperature. The specimens were tested at a rate of extension of $1 \mathrm{~mm} \mathrm{~min} \mathrm{~m}^{-1}$. Five filler volume fractions $v_{f}$ and five 
specimens for each volume fraction were used and the values given correspond to their arithmetic mean value. For the obtention of the stress-strain diagrams strain gauges (KYOWA type, gauge factor $k=1.99$ ) were located on the specimen to measure the strains.

3.1.2. Ultrasonic Experiments. The NDE technique used in the present work was the ultrasonic pulse-echo technique. When ultrasonic pulses are introduced into a specimen, they reflect on a discontinuity or on the back wall of the specimen. The magnitude of the echo reflections depends on the changes in the impedance across the specimen.

To determine the velocities of longitudinal and transverse waves, five specimens from each volume fraction of the composite material were tested ultrasonically at ambient temperature. During each experiment the quantities obtained from the oscilloscope screen were the equivalent thickness $d_{g}$ of the particle-filled composite and the echo heights. Measurements at three different points in each of the five specimens were carried out. From these quantities and using (42), the velocity $c_{\ell}$ was evaluated. A suitable probe for the longitudinal waves with frequency $4 \mathrm{MHz}$ was used. For the evaluation of the velocity $c_{t}$, a suitable probe for the transverse waves with frequency $2 \mathrm{MHz}$ was used. From the analogous (42) this velocity was calculated

$$
C_{t}^{*}=C_{t} \frac{d_{x}}{d_{y}} .
$$

3.2. Dynamic Experiments. The material of the matrix was the same as the material used in the tension experiments and ultrasonic measurements. Four filler volume fractions $(5,10$, 15 , and $20 \%$ ) were used for the study of the effect of filler content on the dynamic properties.

A Dynastat and Dynalyzer apparatus was used for the measurement of the moduli $E_{c}^{\prime}$ and $E_{c}^{\prime \prime}$. This apparatus could apply a sinusoidal load of maximum amplitude $100 \mathrm{~N}$ on a specimen $50 \mathrm{~mm}$ in length and $3.5 \mathrm{~mm}$ wide. The specimen was mounted between a long upper rod connected to a load cell and a short lower rod coupled to a displacement transducer and connected to a motor, which was a coil suspended in the gap of a permanent pole magnet. By passing a servocontrolled current through the coil, the specimen could be subjected to various sinusoidal loads of prescribed amplitude and frequency.

By taking into account the rigidity of the load cell and the type and dimensions of the specimen, the storage and loss moduli were calculated. The measurements were performed at frequencies from 0.1 to $100 \mathrm{~Hz}$ at ambient temperature $\left(20-22^{\circ} \mathrm{C}\right)$.

\section{Results and Discussion}

The 3-component models 1 and 2 were used in [18-20] for the evaluation of the elastic and shear moduli of particulate composites. The proposed models 3 and 4 , as it has been mentioned, are formed from geometrical combinations of the above 3-component models. In Figures 5 and 6 the elastic modulus of the composite is plotted versus the filler volume fraction. In these figures one can also observe the curves corresponding to theoretical values obtained from existing equations in the literature, as well as experimental values obtained from tensile experiments and ultrasonic measurements in epoxy/iron particulate composites. In these figures at first one can observe that the predicted values of $E_{c}$ obtained from models 3 and 4 are bounded from the values predicted from models 1 and 2. In these figures one can also see that the values of $E_{c}$ predicted from model 4 are above those predicted from model 3 . This can be explained by the fact that model 4 consists of more elements from model 1 than model 3 does. In these figures it can also be observed that the predicted values from (12) fit fairly well to the experimental values mainly for high filler volume fraction while the values from (17) are above the experimental values. In Figure 5 it is noticeable that the values predicted by model 3 are close to those obtained by Counto's (A.11) and that they fit more satisfactorily to the experimental values than Counto's. In the same figure it can also be observed that the values from model 2 are close to the values predicted from Kerner (A.3) and (A.4) which are used as a lower bound of the elastic modulus of a particulate composite, and that the values of model 2 are lower than those of Kerner. It is also observed in this figure that (A.5) and (A.6) give lower values than those of (3). Another remark from this figure is that the curves (b), (c), (d), and (e) fit well to the experimental values for low filler volume fraction whereas discrepancies appear for high filler volume fraction. In Figure 6 the theoretical values of $E_{c}$ according to Hashin and Shtrikman bounds are also presented. Comparing the results from Figures 5 and 6 it seems that all the presented curves are bounded by these two curves with little discrepancy in the lower bound. In Figure 6 one can also observe that the values from [16], (A.8), fit to the experimental values for low filler fraction, while discrepancies appear for high filler fraction. This behavior can be explained by the fact that in (A.8) it is assumed that the elastic modulus takes infinite value as $v_{f} \rightarrow 1$. In the same figure one can also see that Narkis (A.7) gives values close to model 3 for the upper bound of this equation. Another point that must be mentioned by comparing these two figures is that the low bound of Hashin and Shtrikman equations gives values close to those calculated from Kerner equations. Finally from these two figures the high discrepancies between theoretical and experimental static results and ultrasonic measurements become obvious. This fact can be attributed to the high frequency that appears in ultrasonic experiments.

The comparison between the theoretical values of $E_{c}$, as they result from models 2, 3, and 4, and the experimental values of $E_{c}$, can be done taking into account the following facts. (a) In [33] it is mentioned that model 1 corresponds to high adhesion quality between matrix and filler and that model 2 corresponds to low adhesion quality while in [34] it is mentioned that models 3 and 4 correspond to an intermediate adhesion quality. (b) In [29] the concept of the interphase is introduced as a third phase whose physicochemical properties assume intermediate values between the values of the corresponding properties of the filler and those of the matrix. The interphase is a zone located 
between the filler and the matrix. In [29] the thickness of the interphase is also evaluated. It is observed that for low filler volume fractions the dependence of the thickness of the interphase upon the filler volume fraction is weak while for higher filler volume fractions this dependence is stronger.

Based upon the above dependence of the interphase thickness upon the filler volume fraction, the observed disagreement between the theoretical and the experimental values of $E_{c}$ can be explained as follows. In the presented models $1,2,3$, and 4 the existence of interphase is not taken into account, but merely a constant adhesion quality is considered for each model, which does not vary with the filler volume fraction. Hence, it is expected that the values of $E_{c}$ furnished by model 3 are higher than the experimental values for low filler volume fractions and are close to the experimental values for higher filler volume fractions. The same remark holds also for models 2 and 4 .

The contribution of models 3 and 4 is that they furnish values of $E_{c}$ lying between the values given by models 1 and 2. These intermediate values can approximate better the experimental values corresponding to higher volume fractions. At the same time the disagreement between the experimental values of $E_{c}$ and the theoretical values, as given by model 3 for low filler volume fractions, can be considered as acceptably small.

It is noticed that the modulus of elasticity given by models 1, 2, 3, and 4 does not depend upon the number of components of each model but upon the geometry of each one. It seems that model 1 and model 2, which are threecomponent models, give an upper bound and a lower bound, respectively, for the values of $E_{c}$ that are evaluated by cubewithin-cube models. Model 4 furnishes higher modulus of elasticity than model 3 because it consists of more geometric elements taken by model 1 , than model 3 does.

In Figure 7 the shear modulus $G_{c}$ in epoxy/iron particulate composite is plotted versus the filler volume fraction. The values of $G_{c}$ predicted from models $1,2,3$, and 4 as well as those evaluated from existing formulae in the literature are presented. The experimental values have been obtained indirectly from tensile experiments by determining simultaneously the elastic modulus and Poisson ratio, and then the shear moduli were calculated from the well-known relation $G=E / 2(1+\nu)$, assuming that the material is macroscopically isotropic. In this figure it can also be observed that the same remarks hold as in the case of uniaxial tensile loading.

In Figure 8 the Poisson ratio values predicted from (23) and from the inverse law of mixtures are plotted versus the filler volume fraction. From this figure it can be seen that the theoretical values predicted by (23) are above those predicted by the inverse law of mixtures, which fit fairly well to the experimental results.

In Figures 9, 10, 11, and 12 the storage modulus $E_{c}^{\prime}$ and the loss modulus $E_{c}^{\prime \prime}$ are plotted versus the filler volume fraction for the frequencies $f=0,1 \mathrm{~Hz}$ and $f=50 \mathrm{~Hz}$, respectively. Although the values of $E_{c}$ predicted by models 3 and 4 approximate better the static experimental results than models 1 and 2, this is not the case for the dynamic experimental results, where the values of $E_{c}^{\prime}$ and $E_{c}^{\prime \prime}$ predicted by model 2 simulate satisfactorily the experimental results. This can be explained by a possible different behavior of the interphase in the static and in the dynamic loading. Assuming that the elastic modulus $E_{i}$ of the interphase varies linearly inside the thickness of the interphase, satisfying the inequality $E_{m} \leq E_{i} \leq E_{f}$, it comes out that the mean value of $E_{i}$ is $\overline{E_{i}}=\left(E_{m}+E_{f}\right) / 2$. Then it seems that models 3 and 4 correspond to a constant value of $E_{i}=\overline{E_{i}}$ inside the thickness of the inerphase, by means of which it comes out that $\varepsilon_{1}=\varepsilon_{2}$ in models 3 and 4 . Inversely, it seems that model 2 corresponds to continuously varying value of $E_{i}$ inside the thickness of the interphase, by means of which it results that $\varepsilon_{1} \neq \varepsilon_{3}$ in model 2. From the above remarks one can conclude that probably the first behavior takes place in the static experiments while the second takes place in the dynamic experiments.

When $m=E_{f} / E_{m} \gg 1$, the dynamic Poisson ratio predicted by (35) leads to (40). One can easily see this, because in $(12)$ and $(17), m /(m-1) \approx 1, m /(m-1) \approx 0$, and the reinforcing coefficients $E_{c} / E_{m}$ and $G_{c} / G_{m}$ are identical. In this case, in epoxy/particle systems, it has been found that the inverse law of mixture simulates satisfactorily the experimental results [25]. For lower values of $m$, deviations of $\nu_{c}$ from the inverse law of mixtures could lead the second member of (38) to be different from zero.

In Figure 13 the storage modulus $E_{c}^{\prime}$ is plotted versus the filler volume fraction. By extending the experimental values of dynamic measurements of the storage modulus $E_{c}^{\prime}$ in epoxy/iron particulate composites, one can reach the values predicted by ultrasonic measurements. This can be explained by the fact that the classification of the local oscillations to oscillations owed to covalent bonds between the atoms of the main chains or to intermolecular bonds probably is not the same for the low and for the high frequencies.

\section{Conclusions}

The values of the elastic and the shear moduli predicted by the new models 3 and 4 are bounded by the values predicted by models 1 and 2 . The values given by model 3 are close to the experimental values mainly for high filler volume fractions, as well as to the values predicted by Counto's equation. The values of $E_{c}$ predicted by this model almost coincide with the average values of $E_{c}$ given by models 1 and 2. Similarly the values of $E_{c}$ given by model 4 fit satisfactorily to the experimental results. Since model 4 is composed by more geometrical elements resulting from model 1 , which is the stiffer model in cube-within-cube formation, it is stiffer than model 3. The values of Poisson ratio resulting from (23) of models (3) and (4) are close to and lower than the values of the Poisson ratio of the matrix. These values have been found to be higher than those predicted by the inverse law of mixtures, which fit fairly well the experimental values.

The dynamic experimental results are approximated satisfactorily by the values of dynamic moduli predicted by model 2. Probably this is due to different behavior of 
the interphase material in static tension than in dynamic loading.

The values of the elastic modulus measured by ultrasonic measurements are higher than those measured by tensile and dynamic experiments. This can be explained by the fact that the number of the local oscillations owed to covalent bonds between the atoms of the main chains is rather greater in higher than in lower frequencies, resulting to an increase of the elastic modulus in high frequencies. Equivalently the number of the local oscillations owed to intermolecular or other bonds weaker than the covalent bonds seems to be lower in higher than in lower frequencies.

\section{Appendices}

\section{A. Elastic Moduli}

The existing models in the literature used for comparison are the following.

(1) Einstein equation

$$
E_{c}=E_{m}\left(1+2,5 v_{f}\right) \text {. }
$$

(2) Equation of Guth and Smallwood

$$
E_{c}=E_{m}\left(1+2,5 v_{f}+14,1 v_{f}^{2}\right)
$$

(3) Kerner equations

$$
\frac{E_{c}}{E_{m}}=\frac{\frac{v_{f} G_{f}}{\left(7-5 v_{m}\right) G_{m}+\left(8-10 v_{m}\right) G_{f}}+\frac{v_{f}}{15\left(1-v_{m}\right)}}{\frac{v_{f} G_{m}}{\left(7-5 v_{m}\right) G_{m}+\left(8-10 v_{m}\right) G_{f}}+\frac{v_{m}}{15\left(1-v_{m}\right)}} .
$$

This equation for $E_{f} \gg E_{m}$ is simplified as follows:

$$
\frac{E_{c}}{E_{m}}=1+\frac{v_{f} \cdot 15\left(1-v_{m}\right)}{v_{m}\left(8-10 v_{m}\right)}
$$

(4) Takahashi equation

$$
\frac{E_{c}}{E_{m}}=1+\left(1-v_{m}\right) v_{f} \frac{E_{f}\left(1-2 v_{m}\right)-E_{m}\left(1-v_{f}\right)+10\left(1+v_{m}\right) E_{f}\left(1+v_{m}\right)-E_{m}\left(1+v_{m}\right)}{E_{f}\left(1+v_{m}\right)+2 E_{m}\left(1-2 v_{f}\right)+2 E_{f}\left(4-5 v_{m}\right)\left(1+v_{m}\right)+E_{m}\left(7-5 v_{m}\right)\left(1+v_{f}\right)}
$$

(5) Equation of Euler and Van Dyck

$$
E_{c}=E_{m}\left(1+\frac{k v_{f}}{1-s v_{f}}\right)
$$

where $k$ and $s$ take the values 1,25 and 1,20, respectively.

(6) Narkis equation

$$
E_{c}=\frac{E_{m}}{k\left(1-v_{f}^{1 / 3}\right)}
$$

where $1,4 \leq k \leq 1,7$.
(7) Mooney equation

$$
E_{c}=E_{m} \exp \frac{1+2,5 v_{f}}{1-5 v_{f}}
$$

where for close sphere packing $s=1,35$.

(8) Hashin and Shtrikman bounds.

The upper and lower bounds are, respectively,

$$
E_{c}=\frac{9\left(K_{m}+\frac{v_{f}}{\frac{1}{K_{f}-K_{m}}+\frac{3 v_{m}}{3 K_{m}+4 G_{m}}}\right)\left(G_{m}+\frac{v_{f}}{\frac{1}{G_{f}-G_{m}}+\frac{6\left(K_{m}+2 G_{m}\right) v_{m}}{5\left(3 K_{m}+4 G_{m}\right) G_{m}}}\right)}{3\left(K_{m}+\frac{v_{f}}{\frac{1}{K_{f}-K_{m}}+\frac{3 v_{m}}{3 K_{m}+4 G_{m}}}\right)+\left(G_{m}+\frac{v_{f}}{\frac{1}{G_{f}-G_{m}}+\frac{6\left(K_{m}+2 G_{m}\right) v_{m}}{5\left(3 K_{m}+4 G_{m}\right) G_{m}}}\right)},
$$




$$
E_{c}=\frac{9\left(K_{f}+\frac{v_{m}}{\frac{1}{K_{m}-K_{f}}+\frac{3 v_{f}}{3 K_{f}+4 G_{f}}}\right)\left(G_{f}+\frac{v_{m}}{\left.\frac{1}{G_{m}-G_{f}}+\frac{6\left(K_{f}+2 G_{f}\right) v_{f}}{5\left(3 K_{f}+4 G_{f}\right) G_{f}}\right)}\right.}{3\left(K_{f}+\frac{v_{m}}{\frac{1}{K_{m}-K_{f}}+\frac{3 v_{f}}{3 K_{f}+4 G_{f}}}\right)+\left(G_{f}+\frac{v_{m}}{\frac{1}{G_{m}-G_{f}}+\frac{6\left(K_{f}+2 G_{f}\right) v_{f}}{5\left(3 K_{f}+4 G_{f}\right) G_{f}}}\right)} .
$$

(9) Counto Model

$$
\frac{1}{E_{c}}=\frac{1-v_{f}^{1 / 2}}{E_{m}}+\frac{1}{\left[\left(1-v_{f}^{1 / 2}\right) / v_{f}^{1 / 2}\right] E_{m}+E_{f}}
$$

\section{B. Storage and Loss Moduli}

The equations of $E_{c}^{\prime}$ and $E_{c}^{\prime \prime}$ used for comparison are the following.

(1) Einstein equation

$$
\begin{aligned}
& E_{c}^{\prime}=E_{m}^{\prime}\left(1+2,5 v_{f}\right), \\
& E_{c}^{\prime \prime}=E_{m}^{\prime \prime}\left(1+2,5 v_{f}\right) .
\end{aligned}
$$

(2) Equation of Guth and Smallwood

$$
\begin{aligned}
& E_{c}^{\prime}=E_{m}^{\prime}\left(1+2,5 v_{f}+14,1 v_{f}^{2}\right), \\
& E_{c}^{\prime \prime}=E_{m}^{\prime \prime}\left(1+2,5 v_{f}+14,1 v_{f}^{2}\right) .
\end{aligned}
$$

(3) Kerner equations

$$
\begin{aligned}
& \frac{E_{c}^{\prime}}{E_{m}^{\prime}}=1+\frac{v_{f} \cdot 15\left(1-v_{m}\right)}{v_{m}\left(8-10 v_{m}\right)}, \\
& \frac{E_{c}^{\prime \prime}}{E_{m}^{\prime \prime}}=1+\frac{v_{f} \cdot 15\left(1-v_{m}\right)}{v_{m}\left(8-10 v_{m}\right)} .
\end{aligned}
$$

(4) Mooney equation

$$
\begin{aligned}
& E_{c}^{\prime}=E_{m}^{\prime} \exp \frac{1+2,5 v_{f}}{1-5 v_{f}}, \\
& E_{c}^{\prime \prime}=E_{m}^{\prime \prime} \exp \frac{1+2,5 v_{f}}{1-5 v_{f}} .
\end{aligned}
$$

\section{References}

[1] P. S. Theocaris, "Viscoelastic properties of epoxy resins derived from creep and relaxation tests at different temperatures," Rheologica Acta, vol. 2, no. 2, pp. 92-96, 1962.

[2] P. S. Theocaris, "Rheologic behavior of epoxy resins in the transition region," Journal of Applied Polymer Science, vol. 8, no. 1, pp. 399-412, 1964.

[3] P. S. Theocaris, "A review of the rheo-optical properties of linear high polymers," Experimental Mechanics, vol. 5, no. 4, pp. 105-114, 1965.

[4] T. Hirai and D. E. Kline, "Dynamic mechanical properties of nonstoichiometric, amine-cured epoxy resin," Journal of Applied Polymer Science, vol. 16, no. 12, pp. 3145-3157, 1972.

[5] T. Hirai and D. E. Kline, "Effects of heat treatment on dynamic mechanical properties of nonstoichiometric, aminecured epoxy resins," Journal of Applied Polymer Science, vol. 17, no. 1, pp. 31-44, 1973.

[6] Z. Hashin, "Complex moduli of viscoelastic composites-I. General theory and application to particulate composites," International Journal of Solids and Structures, vol. 6, no. 5, pp. 539-552, 1970.

[7] P. S. Theocaris and E. Sideridis, "Elastic moduli of particulatefilled polymers," Journal of Applied Polymer Science, vol. 29, no. 10, pp. 2997-3011, 1984.

[8] S. Ahmed and F. R. Jones, "A review of particulate reinforcement theories for polymer composites," Journal of Materials Science, vol. 25, no. 12, pp. 4933-4942, 1990.

[9] A. Einstein, "Investigation of Theory of Brownian Motion," Dover, New York, USA, 1956.

[10] E. Guth, "Theory of filler reinforcement," Journal of Applied Physics, vol. 16, no. 1, pp. 20-25, 1945.

[11] H. M. Smallwood, "Limiting law of the reinforcement of rubber," Journal of Applied Physics, vol. 15, no. 11, pp. 758766, 1944.

[12] E. H. Kerner, "The elastic and thermo-elastic properties of composite media," Proceedings of the Physical Society B, vol. 69, no. 8, pp. 808-813, 1956.

[13] K. Takahashi, M. Ikeda, K. Harakawa, K. Tanaka, and T. Sakai, "Analysis of the effect of interfacial slippage on the elastic moduli of a particle-filled polymer," Journal of Polymer Science: Polymer Physics, vol. 16, no. 3, pp. 415-425, 1978.

[14] H. Eilers, "Die Viskosität von Emulsionen hochviskoser Stoffe als Funktion der Konzentration," Kolloid-Zeitschrift, vol. 97, no. 3, pp. 313-321, 1941. 
[15] M. Narkis, "Crazing in glassy polymers: Studies on polymerglass bead composites," Polymer Engineering and Science, vol. 15 , no. 4, pp. 316-320, 1975.

[16] M. Mooney, "The viscosity of a concentrated suspension of spherical particles," Journal of Colloid Science, vol. 6, no. 2, pp. $162-170,1951$.

[17] Z. Hashin and S. Shtrikman, "A variational approach to the theory of the elastic behaviour of multiphase materials," Journal of the Mechanics and Physics of Solids, vol. 11, no. 2, pp. 127-140, 1963.

[18] B. Paul, "Prediction of Elastic Constants of Multiphase Materials," Transactions of The Metallurgical Society of AIME, vol. 218, pp. 36-41, 1960.

[19] O. Ishai and L. J. Cohen, "Elastic properties of filled and porous epoxy composites," International Journal of Mechanical Sciences, vol. 9, no. 8, pp. 539-546, 1967.

[20] R. M. Ogorckiewicz and G. W. Weidman, "Tensile stiffness of a thermoplastic reinforced with glass fibres or spheres," Journal of Mechanical Engineering Science, vol. 16, no. 1, pp. 10-17, 1974.

[21] M. Takayanagi, S. Uemura, and S. Minami, "Application of equivalent model method to dynamic rheo-optical properties of crystalline polymer," Journal of Polymer Science Part C, vol. 5, no. 1, pp. 113-122, 1964.

[22] U. J. Counto, "The effect of the elastic modulus of the aggregate on the elastic modulus, creep and creep recovery of concrete," Magazine of Concrete Research, vol. 16, no. 48, pp. 129-138, 1964.

[23] R. M. Ogorkiewicz and G. W. Weidmann, "Shear stiffness of epoxy-glass composites," Composites, vol. 5, no. 6, pp. 242 246, 1974.

[24] G. Spathis, G. Bourkas, V. Kytopoulos, and E. Sideridis, "Elastic modulus of particulate composites using a multiphase model," Journal of Reinforced Plastics and Composites, vol. 19, no. 11, pp. 883-910, 2000.

[25] V. N. Kytopoulos, E. Sideridis, and G. D. Bourkas, "A study of some thermomechanical and fractural properties of particle reinforced polymer composites and SEM-aided microfailure approach of certain fracture parameters," Journal of Reinforced Plastics and Composites, vol. 22, no. 17, pp. 1547-1587, 2003.

[26] M. P. Lutz and R. W. Zimmerman, "Effect of the interphase zone on the bulk modulus of a particulate composite," Journal of Applied Mechanics, vol. 63, no. 4, pp. 855-861, 1996.

[27] W. Wang and I. Jasiuk, "Effective elastic constants of participate composites with inhomogeneous interphases," Journal of Composite Materials, vol. 32, no. 15, pp. 1391-1424, 1998.

[28] N. Lombardo, "Effect of an inhomogeneous interphase on the thermal expansion coefficient of a particulate composite," Composites Science and Technology, vol. 65, no. 14, pp. 21182128, 2005.

[29] E. Sideridis, "The dynamic moduli of particulate-filled polymers as defined by the concept of interphase," Composites Science and Technology, vol. 27, no. 4, pp. 305-332, 1986.

[30] P. S. Theocaris, "Interrelation between dynamic moduli and compliances in polymers," Kolloid-Zeitschrift \& Zeitschrift für Polymere, vol. 235, no. 1, pp. 1182-1188, 1969.

[31] S. A. Paipetis, G. Papanicolaou, and P. S. Theocaris, "Dynamic properties of metal-filled epoxy polymers," Fibre Science and Technology, vol. 8, no. 3, pp. 221-242, 1975.

[32] G. Bourkas, V. Kytopoulos, C. Younis, I. Prassianakis, and E. Sideridis, "An estimation of the dynamic moduli and compliances of particulate composites using a simplified approach," in Proceedings of the International Conference on Structural
Analysis of Advanced Materials, Patras, Greece, September 2007.

[33] G. Bourkas, E. Sideridis, C. Younis, I. Prassianakis, and V. Kytopoulos, "Strength and fracture strain of resin/filler systems using two models (1) of perfect and (2) of low adhesion quality," International Journal of Materials and Product Technology. In press.

[34] G. Bourkas, "An intermediate adhesion quality model for the evaluation of strength and inherent flow of resin/filler systems," in Proceedings of the 4th International Conference on Non-Destructive Testing of Hellenic Society of Non-Destructive Testing, Chania, Greece, October 2007. 

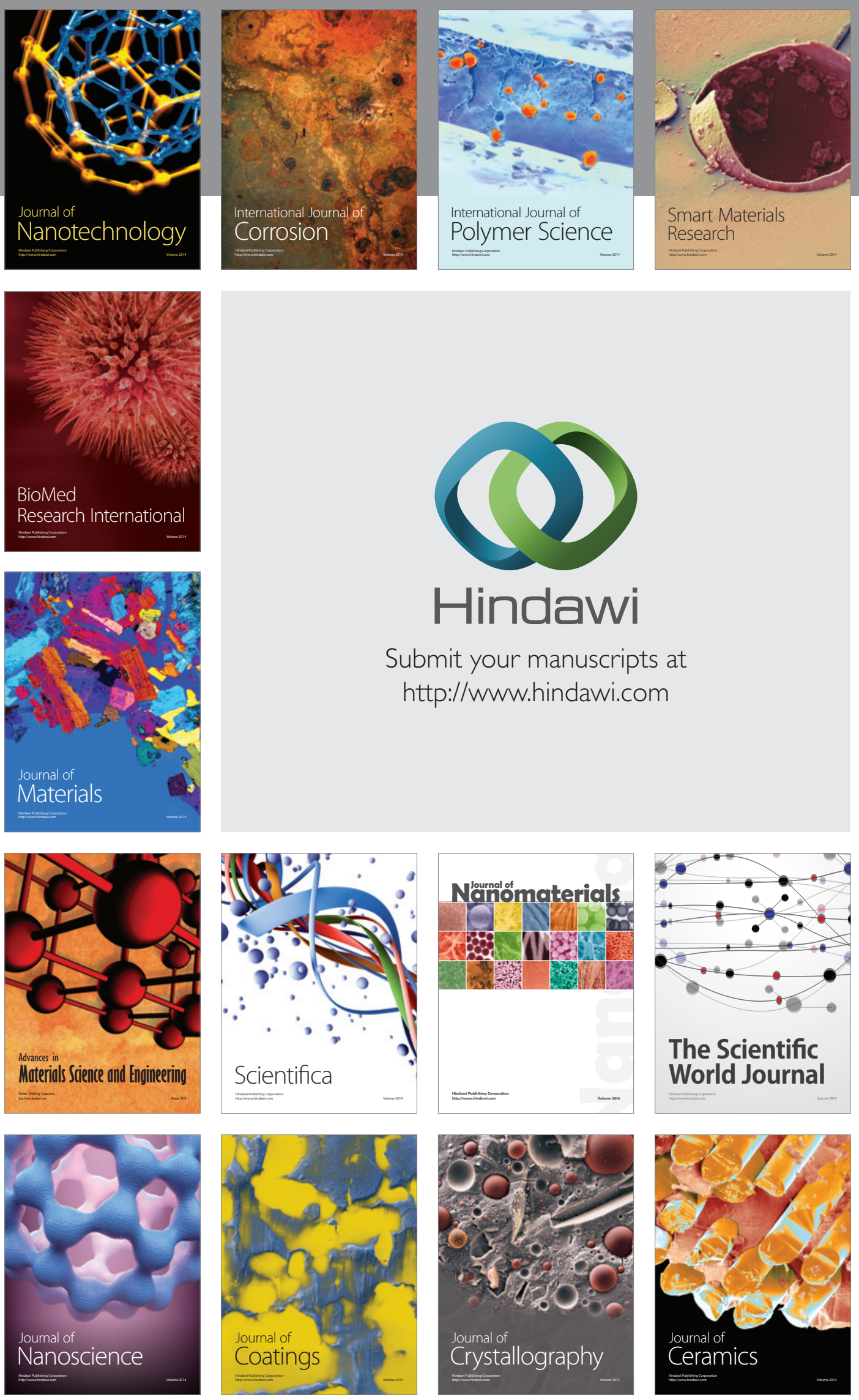

The Scientific World Journal

Submit your manuscripts at

http://www.hindawi.com

\section{World Journal}

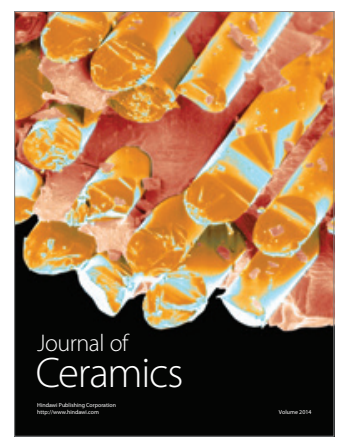

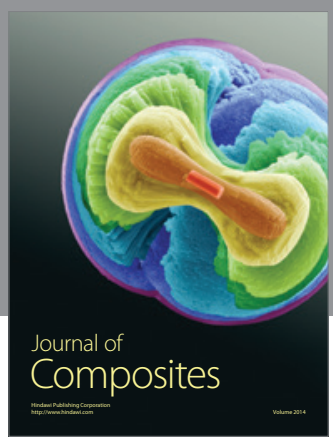
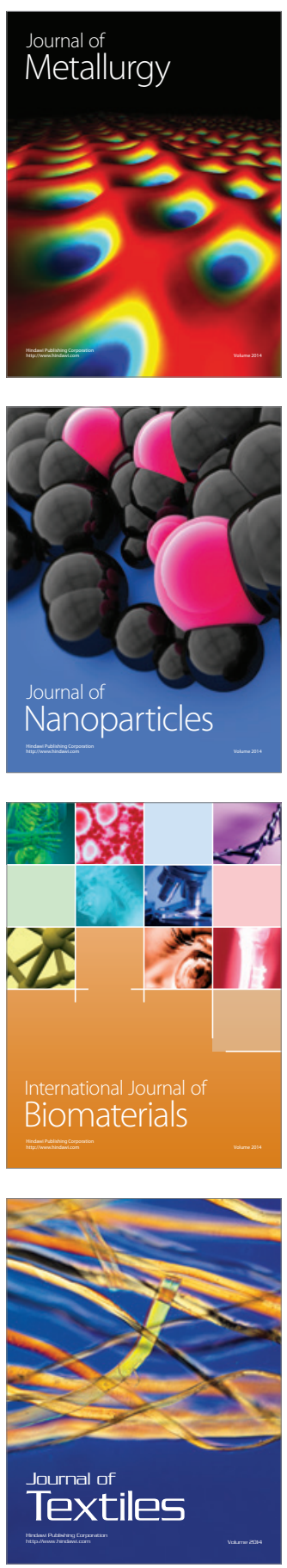